\title{
First detection of triply-deuterated methanol
}

\author{
B. Parise ${ }^{1}$, A. Castets $^{2}$, E. Herbst ${ }^{3}$, E. Caux ${ }^{1}$, C. Ceccarelli ${ }^{4}$, I. Mukhopadhyay ${ }^{5}$, and A. G. G. M. Tielens ${ }^{6}$ \\ 1 CESR CNRS-UPS, BP 4346, 31028 Toulouse Cedex 04, France \\ 2 Observatoire de Bordeaux, BP 89, 33270 Floirac, France \\ 3 Department of Physics, The Ohio State University, 174 W. 18th Ave. Columbus, OH 43210-1106, USA \\ ${ }^{4}$ Laboratoire d'Astrophysique, Observatoire de Grenoble, BP 53, 38041 Grenoble Cedex 09, France \\ 5 Dakota State University, 820 N. Washington Ave., Madison, SD 57042, USA \\ ${ }^{6}$ SRON, PO Box 800, 9700 AV Groningen, The Netherlands
}

Received 7 August 2003 / Accepted 3 November 2003

\begin{abstract}
We report the first detection of triply-deuterated methanol, with 12 observed transitions, towards the low-mass protostar IRAS 16293-2422, as well as multifrequency observations of ${ }^{13} \mathrm{CH}_{3} \mathrm{OH}$, used to derive the column density of the main isotopomer $\mathrm{CH}_{3} \mathrm{OH}$. The derived fractionation ratio $\left[\mathrm{CD}_{3} \mathrm{OH}\right] /\left[\mathrm{CH}_{3} \mathrm{OH}\right]$ averaged on a $10^{\prime \prime}$ beam is $1.4 \%$. Together with previous $\mathrm{CH}_{2} \mathrm{DOH}$ and $\mathrm{CHD}_{2} \mathrm{OH}$ observations, the present $\mathrm{CD}_{3} \mathrm{OH}$ observations are consistent with a formation of methanol on grain surfaces, if the atomic $\mathrm{D} / \mathrm{H}$ ratio is 0.1 to 0.3 in the accreting gas. Such a high atomic ratio can be reached in the framework of gas-phase chemical models including all deuterated isotopomers of $\mathrm{H}_{3}^{+}$.
\end{abstract}

Key words. ISM: abundances - ISM: molecules - stars: formation - ISM: individual: IRAS 16293-2422

\section{Introduction}

Despite the relatively low elemental abundance of deuterium in space (a factor of $\sim 1.5 \times 10^{-5}$ less abundant than $\mathrm{H}$; Linsky 1998), extremely large amounts of doubly-deuterated formaldehyde $\left(\mathrm{D}_{2} \mathrm{CO} / \mathrm{H}_{2} \mathrm{CO} \sim 10 \%\right)$ have been observed in the solar-type protostar IRAS 16293-2422 (hereafter IRAS 16293, Ceccarelli et al. 1998; Loinard et al. 2000; Ceccarelli et al. 2001), initiating the search for other multiply deuterated molecules. Subsequently, doubly deuterated formaldehyde, doubly deuterated hydrogen sulfide and multiply deuterated ammonia have been observed in other protostars and dark clouds from where protostars form (Roueff et al. 2000; Loinard et al. 2001; Ceccarelli et al. 2002; van der Tak et al. 2002; Lis et al. 2002; Vastel et al. 2003). These studies have been interpreted in terms of two different routes for formaldehyde, hydrogen sulfide and ammonia deuteration: active grain chemistry followed by at least partial desorption into the gas for formaldehyde and hydrogen sulfide on the one hand and gas-phase chemistry for ammonia on the other hand. However, ammonia may also be a grain surface product, provided a large $\mathrm{D} / \mathrm{H}$ atomic ratio in the accreting gas. Recently, doubly-deuterated methanol was detected towards IRAS 16293 (Parise et al. 2002). This observation provided new constraints for chemical models. The observations of the deuterated methanols $\mathrm{CH}_{2} \mathrm{DOH}$ and $\mathrm{CHD}_{2} \mathrm{OH}$ were both consistent with the formation of methanol from successive hydrogenations of $\mathrm{CO}$ by reaction with atomic $\mathrm{H}$ on

Send offprint requests to: B. Parise, e-mail: Berengere.Parise@cesr.fr grain surfaces, but required an atomic $\mathrm{D} / \mathrm{H}$ ratio of 0.2 to 0.3 in the accreting gas. At the time of the observation of doublydeuterated methanol, no gas-phase model was able to predict such a high atomic $\mathrm{D} / \mathrm{H}$ ratio. Meantime, observations of doubly deuterated formaldehyde in a sample of pre-stellar cores showed that the degree of deuteration increases with increasing CO depletion (Bacmann et al. 2002, 2003). This deuteration of formaldehyde in pre-stellar cores may occur partially in the CO-depleted gas-phase and partially on the surface of dust grains, followed by some inefficient desorption mechanism. A further spectacular confirmation of enhanced deuteration in CO-depleted gas came from the detection of abundant $\mathrm{H}_{2} \mathrm{D}^{+}$, likely the most abundant ion, in the prestellar core L1544 (Caselli et al. 2003). Phillips \& Vastel (2003) suggested that in CO-depleted gas, even the multiple deuterated forms of $\mathrm{H}_{3}^{+}$may be abundant and play a role in the molecular deuteration enhancement. The suggestion has been fully confirmed by the modelling of Roberts et al. (2003), which shows that including $\mathrm{HD}_{2}^{+}$and $\mathrm{D}_{3}^{+}$in the chemical network increases dramatically the molecular deuteration, and allows the production of the large atomic $\mathrm{D} / \mathrm{H}$ ratio predicted by the methanol observations (Parise et al. 2002).

In this paper, we report the first detection of triplydeuterated methanol $\mathrm{CD}_{3} \mathrm{OH}$ in space, performed towards the solar-type protostar IRAS 16293 . We also present a multifrequency observation of ${ }^{13} \mathrm{CH}_{3} \mathrm{OH}$, used to derive the column density of the main isotopomer $\mathrm{CH}_{3} \mathrm{OH}$. These observations provide yet another stringent test to confirm the validity of grain surface models. 
Table 1. Main-beam intensities ${ }^{1}$, peak temperatures ${ }^{2}$ and widths for the observed $\mathrm{CD}_{3} \mathrm{OH}$ and ${ }^{13} \mathrm{CH}_{3} \mathrm{OH}$ transitions ${ }^{3}$.

\begin{tabular}{|c|c|c|c|c|c|c|}
\hline $\begin{array}{c}\text { Frequency } \\
\mathrm{GHz}\end{array}$ & Transition & $\begin{array}{c}\mu^{2} S \\
\text { Debye }^{2} \\
\end{array}$ & $\begin{array}{c}E_{\text {up }} \\
\mathrm{K}\end{array}$ & $\begin{array}{c}\int T_{\mathrm{mb}} \mathrm{d} v \\
\mathrm{~K} \mathrm{~km} \mathrm{~s}^{-1}\end{array}$ & $\begin{array}{l}T_{\mathrm{mb}} \\
\mathrm{mK}\end{array}$ & $\begin{array}{c}\Delta v \\
\mathrm{~km} \mathrm{~s}^{-1}\end{array}$ \\
\hline \multicolumn{7}{|l|}{$\mathrm{CD}_{3} \mathrm{OH}$} \\
\hline $156.237016^{\star}$ & $4_{1}-3_{1} E_{2}$ & 2.94 & 21.5 & $0.102 \pm 0.023$ & 51 & $1.8 \pm 0.4$ \\
\hline 156.239295 & $4_{2}-3_{2} \mathrm{~A}-$ & 2.44 & 42.0 & $0.032 \pm 0.012$ & 26 & $1.2 \pm 0.5$ \\
\hline 156.242613 & $4_{0}-3_{0} A+$ & 3.13 & 18.8 & $0.059 \pm 0.018$ & 31 & $1.8 \pm 0.5$ \\
\hline 156.253079 & $4_{2}-3_{2} A+$ & 2.44 & 42.0 & $0.056 \pm 0.015$ & 38 & $1.4 \pm 0.4$ \\
\hline $156.260737^{\star}$ & $4_{3}-3_{3} E_{2}$ & 1.39 & 55.5 & $0.061 \pm 0.018$ & 46 & $1.3 \pm 0.4$ \\
\hline 156.262936 & $4_{3}-3_{3} E_{1}$ & 1.37 & 46.9 & $0.044 \pm 0.011$ & 43 & $1.0 \pm 0.2$ \\
\hline 156.275238 & $4_{1}-3_{1} E_{1}$ & 2.93 & 33.1 & $0.034 \pm 0.021$ & 26 & $1.2 \pm 1.2$ \\
\hline $156.285288^{\star}$ & $4_{2}-3_{2} E_{2}$ & 2.35 & 36.3 & $0.065 \pm 0.021$ & 54 & $1.1 \pm 0.3$ \\
\hline 156.581519 & $8{ }_{1}-7_{0} \mathrm{E}_{2}$ & 4.15 & 70.2 & $0.050 \pm 0.010$ & 46 & $1.0 \pm 0.2$ \\
\hline 160.640122 & $2{ }_{0}-2_{1} E_{2}$ & 2.38 & 15.95 & $0.090 \pm 0.019$ & 26 & $3.2 \pm 0.5$ \\
\hline 160.718291 & $6_{2}-5_{1} E_{1}$ & 2.22 & 50.2 & $0.039 \pm 0.009$ & 29 & $1.3 \pm 0.2$ \\
\hline 160.753934 & $1_{0}-1_{1} E_{2}$ & 1.45 & 12.2 & $0.038 \pm 0.009$ & 35 & $1.0 \pm 0.2$ \\
\hline \multicolumn{7}{|l|}{${ }^{13} \mathrm{CH}_{3} \mathrm{OH}$} \\
\hline 156.299374 & $5_{05}-5_{-15}$ & 0.697 & 47.1 & $0.19 \pm 0.08$ & 65 & $3.0 \pm 0.5$ \\
\hline 160.507694 & $2_{12}-3_{03}$ & 0.300 & 21.3 & $0.08 \pm 0.02$ & 29 & $2.4 \pm 0.6$ \\
\hline 330.194042 & $7_{-17}-6_{-16}$ & 5.55 & 69.0 & $0.51 \pm 0.16$ & 120 & $3.8 \pm 0.6$ \\
\hline 330.252798 & $7_{07}-6_{06}$ & 5.66 & 63.4 & $0.43 \pm 0.15$ & 100 & $4.0 \pm 1.2$ \\
\hline 330.265233 & $7-61-6_{-60}$ & 1.50 & 253.4 & $0.15 \pm 0.10$ & 80 & $1.7 \pm 1.0$ \\
\hline 330.277270 & $7_{61}-6_{60}$ & 1.50 & 258.1 & $0.12 \pm 0.08$ & 60 & $2.0 \pm 0.7$ \\
\hline 330.277270 & $7_{62}-6_{61}$ & 1.50 & 258.1 & $0.12 \pm 0.08$ & 60 & $2.0 \pm 0.7$ \\
\hline 330.319110 & $7_{52}-6_{51}$ & 2.78 & 202.0 & $0.45 \pm 0.21$ & 90 & $4.8 \pm 0.9$ \\
\hline 330.319110 & $7_{53}-6_{52}$ & 2.78 & 202.0 & $0.45 \pm 0.21$ & 90 & $4.8 \pm 0.9$ \\
\hline 330.342534 & $7_{44}-6_{43}$ & 3.81 & 144.2 & $0.07 \pm 0.05$ & 50 & $1.3 \pm 6.7$ \\
\hline 330.342534 & $7_{43}-6_{42}$ & 3.81 & 144.2 & $0.07 \pm 0.05$ & 50 & $1.3 \pm 6.7$ \\
\hline 330.408395 & $7_{34}-6_{33}$ & 4.61 & 111.4 & $0.91 \pm 0.19$ & 200 & $4.2 \pm 0.7$ \\
\hline 330.442421 & $7_{16}-6_{15}$ & 5.69 & 84.49 & $0.42 \pm 0.12$ & 140 & $2.8 \pm 0.9$ \\
\hline 330.535822 & $7_{25}-6_{24}$ & 5.14 & 85.80 & $0.25 \pm 0.11$ & 40 & $5.6 \pm 1.2$ \\
\hline 330.535890 & $7-26-6-25$ & 5.20 & 89.45 & $0.25 \pm 0.11$ & 40 & $5.6 \pm 1.2$ \\
\hline
\end{tabular}

${ }^{1}$ The fluxes were derived using Gaussian fits, and the uncertainty given is $\sqrt{\sigma_{\text {stat }}^{2}+\sigma_{\text {cal }}^{2}}$ where $\sigma_{\text {stat }}$ is the statistical error and $\sigma_{\text {cal }}$ the calibration uncertainty (15\%). ${ }^{2}$ The noise rms is $8 \mathrm{mK}$ for the $\mathrm{CD}_{3} \mathrm{OH}$ data and $37 \mathrm{mK}$ for the ${ }^{13} \mathrm{CH}_{3} \mathrm{OH}$ data. ${ }^{3} \mathrm{~A}$ star following the frequency indicates that the line is close to a $\mathrm{CH}_{2} \mathrm{DCN}$ line and was fitted by a two-component Gaussian fit (see text).

\section{Observations and results}

Using the IRAM 30-meter telescope (Pico Veleta, Spain), we detected the $12 \mathrm{CD}_{3} \mathrm{OH}$ lines reported in Table 1 . The telescope was pointed at the coordinates $\alpha(2000)=16^{\mathrm{h}} 32^{\mathrm{m}} 22.6^{\mathrm{s}}$ and $\delta(2000)=-24^{\circ} 28^{\prime} 33.0^{\prime \prime}$. The observations were performed in April 2003. Two receivers were used simultaneously at $2 \mathrm{~mm}$, to observe two bands around 156 and $160 \mathrm{GHz}$, with typical system temperatures of about 230 and $250 \mathrm{~K}$ respectively. These receivers were connected to the VESPA autocorrelator divided in six units. The telescope beam width is approximately $15^{\prime \prime}$ at $160 \mathrm{GHz}$. All observations were performed using the wobbler switching mode with an OFF position 4' from the source. The pointing accuracy was monitored regularly on strong continuum sources, and was found to be better than $3^{\prime \prime}$. All spectra were obtained with an integration time of $750 \mathrm{~min}$. The rms noise is equal to $8 \mathrm{mK}\left(T_{\mathrm{mb}}\right)$ for a spectral resolution of $0.3 \mathrm{~km} \mathrm{~s}^{-1}$.
Observed spectra are shown in Fig. 1. The measured intensities, linewidths and main-beam temperatures are reported in Table 1. The frequencies of all detected lines have previously been measured in the laboratory with an accuracy of $25 \mathrm{kHz}$ (Walsh et al. 1998), while the transition strengths and energy levels were estimated from the published spectroscopic constants (Predoi-Cross et al. 1998) using the methanol program at Ohio State.

Some of the $\mathrm{CD}_{3} \mathrm{OH}$ lines (indicated by a star in Table 1) are close to $\mathrm{CH}_{2} \mathrm{DCN}$ lines. In that case, the intensity was derived by using a two-component Gaussian fit, so the quoted fluxes have a further uncertainty due to the relative line contribution.

Two ${ }^{13} \mathrm{CH}_{3} \mathrm{OH}$ lines at $156 \mathrm{GHz}$ were observed simultaneously to the $\mathrm{CD}_{3} \mathrm{OH}$ lines. In addition, we analysed $330 \mathrm{GHz}{ }^{13} \mathrm{CH}_{3} \mathrm{OH}$ observations obtained using the JCMT in January 2000, with an rms noise of $37 \mathrm{mK}$. The beam size of the JCMT is $15^{\prime \prime}$ at the considered frequencies, i.e. equivalent 

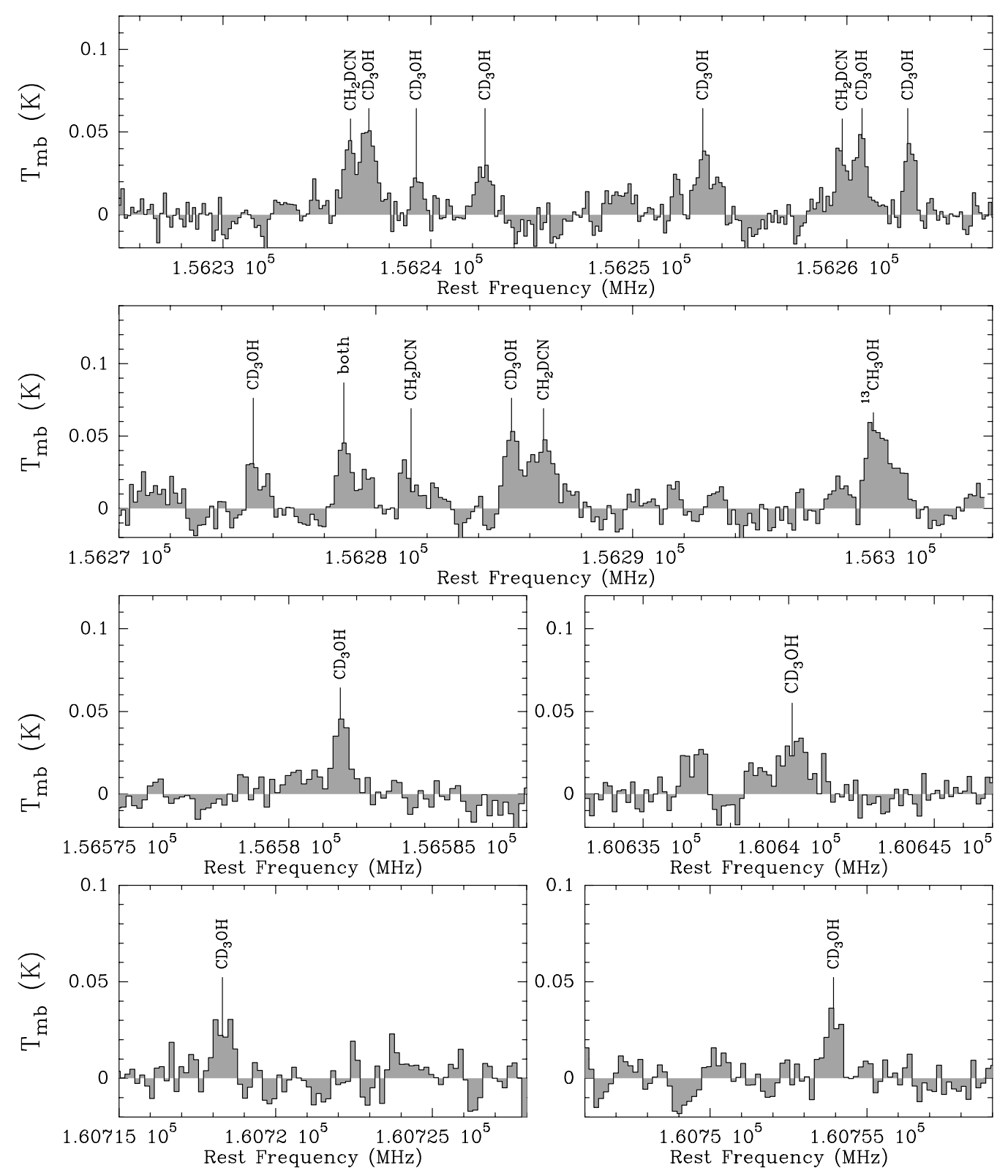

Fig. 1. $\mathrm{CD}_{3} \mathrm{OH}$ detected lines. The intensities are reported in main-beam brightness temperature. The label "both" indicates the blending of one $\mathrm{CD}_{3} \mathrm{OH}$ and one $\mathrm{CH}_{2} \mathrm{DCN}$ lines. This latter $\mathrm{CD}_{3} \mathrm{OH}$ line has not been considered in the population diagram analysis.

to the beam size of the 30-meter at $160 \mathrm{GHz}$. Detailed information concerning the ${ }^{13} \mathrm{CH}_{3} \mathrm{OH}$ spectra is presented in Table 1.

\section{Derivation of the column densities}

We derived the abundance of $\mathrm{CD}_{3} \mathrm{OH}$ using the method of rotational diagrams. The $\mathrm{A}$ and $\mathrm{E}$ species are considered to be linked by ion-molecule reactions that transfer molecules from one species to the other. We then computed one single rotational diagram for the two species, presented in Fig. 2a. We averaged the level column densities on a $10^{\prime \prime}$ beam, as in Parise et al. (2002), following the suggestion by van Dishoeck et al. (1995) of enhanced methanol emission in the central 10" region of IRAS 16293. A more recent study of the spatial distribution of $\mathrm{CH}_{3} \mathrm{OH}$ was performed by Schoier et al. (2002), and showed evidence for an abundance jump of methanol of two orders of magnitude in the inner part of the envelope ( $\leq 150 \mathrm{AU})$. However, in the following we consider averaged abundances on a $10^{\prime \prime}$ beam for consistency with the Parise et al. (2002) study.

The ground $\mathrm{E}$ state is estimated to lie about $4.6 \mathrm{~K}$ above the ground A state. An A state has a relative spin-torsional weight of 11 whereas the relative spin-torsional weight of an $E$ state is 16. The partition function was computed from the asymmetrictop approximation:

$Z(T)=11 \times Z_{\mathrm{A}}(T)+16 \times \exp (-4.6 / T) \times Z_{\mathrm{E}}(T)$

where $Z_{\mathrm{A}}(T)=Z_{\mathrm{E}}(T)=\sqrt{\frac{\pi T^{3}}{A B C}}$, with $A=3.3957 \mathrm{~K}, B=$ $0.9529 \mathrm{~K}$ and $C=0.9247 \mathrm{~K}$, as determined by Walsh et al. (1998).

By fitting a straight line to the data in the rotational diagram, we derive a rotational temperature of $85 \pm 28 \mathrm{~K}$, 

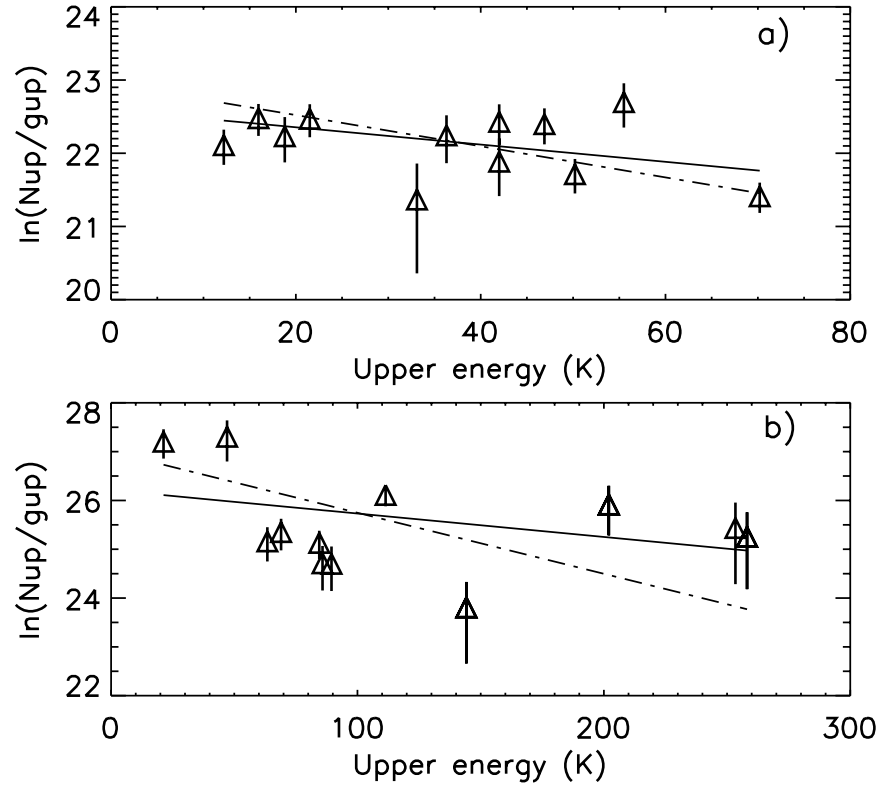

Fig. 2. Rotational diagram for: a) $\mathrm{CD}_{3} \mathrm{OH}$ (solid line $T_{\text {rot }}=85 \mathrm{~K}$, dashed line: $T_{\text {rot }}=47 \mathrm{~K}$, see text), b) ${ }^{13} \mathrm{CH}_{3} \mathrm{OH}$ (solid line: $T_{\text {rot }}=$ $208 \mathrm{~K}$, dashed line: $T_{\text {rot }}=80 \mathrm{~K}$, see text). Column densities are averaged on a $10^{\prime \prime}$ beam.

Table 2. Derived column densities and fractionation ratios relative to $\mathrm{CH}_{3} \mathrm{OH}$ for deuterated methanols in IRAS 16293 .

\begin{tabular}{cccc}
\hline \hline Molecule & $T_{\text {rot }}(\mathrm{K})$ & $N\left(\mathrm{~cm}^{-2}\right)$ & fractionation \\
$\mathrm{CD}_{3} \mathrm{OH}$ & $85 \pm 28$ & $(1.4 \pm 0.9) \times 10^{14}$ & $1.4 \pm 1.4 \%$ \\
& $47 \pm 7^{a}$ & $(7.8 \pm 2.3) \times 10^{13}$ & $0.8 \pm 0.6 \%$ \\
$\mathrm{CHD}_{2} \mathrm{OH}^{b}$ & $47 \pm 7$ & $(6.0 \pm 2.2) \times 10^{14}$ & $6 \pm 5 \%$ \\
$\mathrm{CH}_{2} \mathrm{DOH}^{b}$ & $48 \pm 3$ & $(3.0 \pm 0.6) \times 10^{15}$ & $30 \pm 20 \%$ \\
$\mathrm{CH}_{3} \mathrm{OD}^{b}$ & $20 \pm 4$ & $(1.5 \pm 0.7) \times 10^{14}$ & $2 \pm 1 \%$ \\
\hline
\end{tabular}

${ }^{a}$ Fixed temperature, see text. ${ }^{b}$ Observed in Parise et al. (2002).

consistent with the rotational temperature of $\mathrm{CH}_{3} \mathrm{OH}$ (van Dishoeck et al. 1995). The $\mathrm{CD}_{3} \mathrm{OH}$ column density is $(1.4 \pm 0.9) \times 10^{14} \mathrm{~cm}^{-2}$. We also derived the $\mathrm{CD}_{3} \mathrm{OH}$ column density by fixing the rotational temperature to the one inferred from the $\mathrm{CH}_{2} \mathrm{DOH}$ and $\mathrm{CHD}_{2} \mathrm{OH}$ molecules $\left(T_{\text {rot }}=\right.$ $47 \pm 7 \mathrm{~K}$, Parise et al. 2002). The $\mathrm{CD}_{3} \mathrm{OH}$ column density is then $(7.8 \pm 2.3) \times 10^{13} \mathrm{~cm}^{-2}$. Table 2 lists the column densities for all deuterated methanols observed in IRAS 16293.

The column density of ${ }^{13} \mathrm{CH}_{3} \mathrm{OH}$ was derived using the same method, with the molecular parameters taken from the Cologne Database for Molecular Spectroscopy (Muller et al. 2001). The rotational diagram is presented in Fig. 2b. The inferred rotational temperature is $208 \pm 70 \mathrm{~K}$ and the column density averaged over a $10^{\prime \prime}$ beam is $(2.6 \pm 1.8) \times 10^{14} \mathrm{~cm}^{-2}$. We also computed the column density for fixed rotational temperatures of $50 \mathrm{~K}$ and $80 \mathrm{~K}$. The inferred value is $(1.4 \pm$ 0.6) $\times 10^{14} \mathrm{~cm}^{-2}$, independent of the temperature in this range. Using the ${ }^{12} \mathrm{C} /{ }^{13} \mathrm{C}$ ratio of 70 derived by Boogert et al. (2002), we derive a column density of $(9.8 \pm 4.2) \times 10^{15} \mathrm{~cm}^{-2}$ for $\mathrm{CH}_{3} \mathrm{OH}$. This value is nearly 3 times higher than the column density used by Parise et al. (2002), inferred from $\mathrm{CH}_{3} \mathrm{OH}$ observations from van Dishoeck et al. (1995). The fractionation ratios, relative to this new estimate of the $\mathrm{CH}_{3} \mathrm{OH}$ column density, are reported in Table 2 for all deuterated isotopomers of methanol.

\section{Discussion and conclusions}

The main result of this Paper is the first detection of triplydeuterated methanol in space, with 12 detected transitions. This discovery follows the detection of doubly-deuterated as well as singly-deuterated isotopomers towards the same object (Parise et al. 2002). Observations of multiple isotopomers of methanol represent a powerful constraining tool for chemical processes that lead to such a high deuteration.

It is interesting to compare these observations to the predictions of the simple grain chemistry scheme of Rodgers \& Charnley (2002). If the D atoms are randomly distributed in the methanol isotopomers (i.e. this scheme does not consider any activation barrier for the reactions but rather assumes that all reactions are equiprobable), the fractionation ratios $R$ of each isotopomer relative to $\mathrm{CH}_{3} \mathrm{OH}$ should scale as follows: $R\left(\mathrm{CH}_{3} \mathrm{OD}\right)=\alpha, R\left(\mathrm{CH}_{2} \mathrm{DOH}\right)=3 \alpha, R\left(\mathrm{CHD}_{2} \mathrm{OH}\right)=$ $3 \alpha^{2}$ and $R\left(\mathrm{CD}_{3} \mathrm{OH}\right)=\alpha^{3}$, where $\alpha$ is the accreting atomic D over $\mathrm{H}$ ratio. The three independent observations of $\mathrm{CH}_{2} \mathrm{DOH}$, $\mathrm{CHD}_{2} \mathrm{OH}$ and $\mathrm{CD}_{3} \mathrm{OH}$ are consistent within the error bars with a value of $0.1-0.2$ for the $\mathrm{D}$ over $\mathrm{H}$ accretion rate. Accounting for the different mass of the atoms, this ratio corresponds to an abundance ratio in the gas-phase of $\mathrm{D} / \mathrm{H}=\sqrt{2} \times(0.1-0.2)=$ $0.15-0.3$. However, this simple scheme fails to explain the observed low abundance of $\mathrm{CH}_{3} \mathrm{OD}$.

More accurate grain chemical models accounting for different activation barriers for the reactions have been developed in the last few years. We compare in the following our observations with the model developed by Stantcheva \& Herbst (2003). This model is based on the direct solution of the master equation and therefore gives essentially the same predictions as the Monte Carlo models described by Caselli et al. (2002) or Charnley et al. (1997). Figure 3 shows predictions for fractionation ratios of deuterated isotopomers of methanol relative to $\mathrm{CH}_{3} \mathrm{OH}$ versus the atomic $\mathrm{D} / \mathrm{H}$ ratio in the accreting gas (Stantcheva \& Herbst 2003) when the various isotopomers are formed by active grain chemistry. In the limit of low temperature $(10 \mathrm{~K})$, this model essentially gives the ratios corresponding to a random distribution of deuterium atoms. Observed fractionation ratios with their error bars have been overlaid on each curve, allowing the derivation of the required atomic $\mathrm{D} / \mathrm{H}$ ratio in the gas-phase. The $\mathrm{CD}_{3} \mathrm{OH}, \mathrm{CHD}_{2} \mathrm{OH}$ and $\mathrm{CH}_{2} \mathrm{DOH}$ observations are consistent with a formation on grain surfaces with an atomic $\mathrm{D} / \mathrm{H}$ abundance ratio of $0.1-0.2$. Such a high atomic fractionation ratio in the gas phase is predicted by the recent gas-phase model of Roberts et al. (2003), which involves not only $\mathrm{H}_{2} \mathrm{D}^{+}$but also $\mathrm{D}_{2} \mathrm{H}^{+}$and $\mathrm{D}_{3}^{+}$as precursors for deuterium fractionation, when the density of gas is very high and heavy species such as $\mathrm{CO}$ are strongly depleted.

As can be seen in Fig. 3, $\mathrm{CH}_{3} \mathrm{OD}$ appears to be underdeuterated when compared with the grain chemical predictions. It is possible that the $\mathrm{CH}_{3} \mathrm{OD}$ fractionation may be affected in the warm gas; e.g., this isotopomer may be preferentially converted into $\mathrm{CH}_{3} \mathrm{OH}$ when released in the gas-phase by 


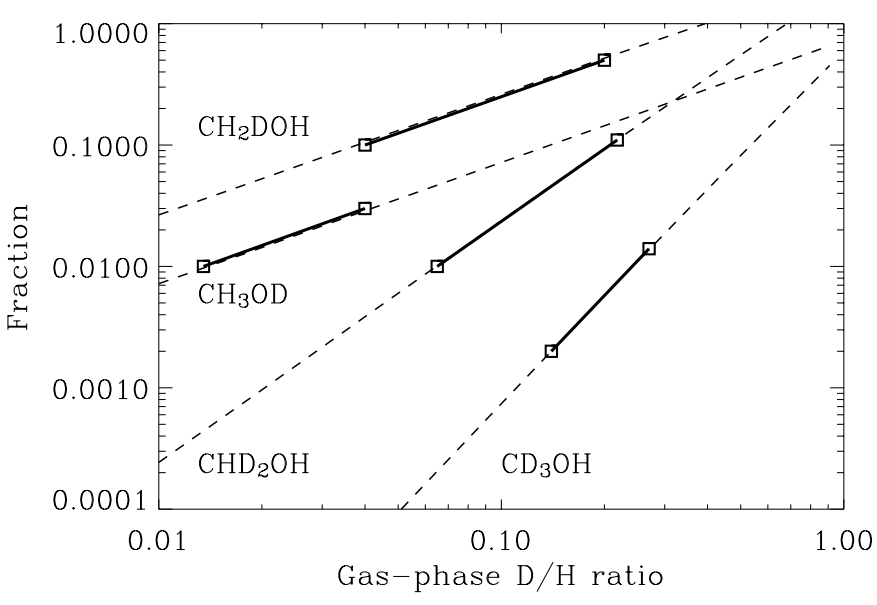

Fig. 3. The deuterium fractionation ratio for the various deuterated isotopomers of methanol is plotted against the abundance ratio of deuterium to hydrogen atoms in the gas phase. Dashed lines: model results of Stantcheva et al. (2003). Thick lines: observations of IRAS 16293.

protonation reactions followed by dissociative recombination with an electron (Charnley et al. 1997; Parise et al. 2002):

$\mathrm{CH}_{3} \mathrm{OD}+\mathrm{H}_{3}^{+} \rightarrow \mathrm{CH}_{3} \mathrm{ODH}^{+}+\mathrm{H}_{2}$

$\mathrm{CH}_{3} \mathrm{OHD}^{+}+\mathrm{e}^{-} \rightarrow \mathrm{CH}_{3} \mathrm{OH}+\mathrm{D}$.

The corresponding reactions with $\mathrm{H}_{2} \mathrm{D}^{+}, \mathrm{HD}_{2}^{+}$and $\mathrm{D}_{3}^{+}$are of little importance in view of their low abundance in the warm gas of the hot core. This hypothesis, which assumes that protonation reactions attack the oxygen end of the methanol only (Osamura et al. in prep.), could be tested by observing the $\mathrm{CH}_{2}$ DOD isotopomer. This observation may be difficult due to the expected low intensity of the lines.

Acknowledgements. E. Herbst acknowledges the support of the National Science Foundation (US) for his research program in astrochemistry.

\section{References}

Bacmann, A., Lefloch, B., Ceccarelli, C., et al. 2002, A\&A, 389, L6 Bacmann, A., Lefloch, B., Ceccarelli, C., et al. 2003, ApJ, 585, L55
Boogert, A. C. C., Blake, G. A., \& Tielens, A. G. G. M. 2002, ApJ, 577, 271

Caselli, P., Stantcheva, T., Shalabiea, O., et al. 2002, Planet. Space Sci., 50, 1257

Caselli, P., van der Tak, F. F. S., Ceccarelli, C., \& Bacmann, A. 2003, A\&A, 403, L37

Cazaux, S., Tielens, A. G. G. M., Ceccarelli, C., et al. 2003, ApJL, in press

Ceccarelli, C., Vastel, C., Tielens, A. G. G. M., et al. 2002, A\&A, 381, L17

Ceccarelli, C., Loinard, L., Castets, A., et al. 2001, A\&A, 372, 998

Ceccarelli, C., Castets, A., Loinard, L., Caux, E., \& Tielens, A. G. G. M. 1998, A\&A, 338, L43

Charnley, S. B., Tielens, A. G. G. M., \& Rodgers, S. D. 1997, ApJ, 482, L203

Linsky, J. L. 1998, Space Sci. Rev., 84, 285

Lis, D. C., Roueff, E., Gerin, M., et al. 2002, ApJ, 571, L55

Loinard, L., Castets, A., Ceccarelli, C., Caux, E., \& Tielens, A. G. G. M. 2001, ApJ, 552, L163

Loinard, L., Castets, A., Ceccarelli, C., et al. 2000, A\&A, 359, 1169

Maret, S., et al. in preparation

Muller, H. S. P., Thorwirth, S., Roth, D. A., \& Winnewisser, G. 2001, A\&A, 370, L49

Parise, B., Ceccarelli, C., Tielens, A. G. G. M., et al. 2002, A\&A, 393, L49

Phillips, T., \& Vastel, C. 2003, in Chemistry as a Diagnostic of Star Formation, ed. C. L. Curry \& M. Fish, in press [astro-ph/0211610]

Predoi-Cross, A., Xu. L.-H., Walsh, M. S., et al.. 1998, J. Mol. Spec., 188, 94

Roberts, H., Herbst, E., \& Millar, T. J. 2003, ApJ, 591, 41

Rodgers, S. D., \& Charnley, S. B. 2002, Planet. Space Sci., 50, 1125

Roueff, E., Tiné, S., Coudert, L. H., et al. 2000, A\&A, 354, L63

Schoier, F. L., Jorgensen, J. K., van Dishoeck, E. F., \& Blake, G. A. 2002, A\&A, 390, 1001

Stantcheva, T., \& Herbst, E. 2003, MNRAS, 340, 983

van der Tak, F. F. S., Schilke, P., Müller, H. S. P., et al. 2002, A\&A, 388, L53

van Dishoeck, E. F., Blake, G. A., Jansen, D. J., \& Groesbeck, T. D. 1995, ApJ, 447, 760

Vastel, C., Phillips, T. G., Ceccarelli, C., \& Pearson, J. 2003, ApJ, 593, L97

Walsh, M. S., Xu, L.-H., \& Lees, R. M. 1998, J. Mol. Spectrosc., 188, 85 